\title{
Fallas Humanas y Accidentes de Trabajo: Una Contribución de las Investigaciones en Prevención, Control de los Riesgos y Accidentes
}

\section{HUMAN ERRORS AND ACCIDENTS AT WORK: CONTRIBUTION OF ACCIDENT INVESTIGATIONS IN THE PREVENTION AND CONTROL OF RISKS}

\author{
Fabiano Takeda', Antônio Renato Pereira Moro', Ana M Gonzales', Armando Monterrosa Quintero² \\ 1. Centro Tecnológico - CTC, Universidad Federal de Santa Catarina, Brasil. \\ 2. Centro Deportivo - CDS, Universidad Federal de Santa Catarina, Brasil.
}

\section{RESUMEN}

Con el objetivo de contribuir en el control de accidentes de trabajo, esta investigación busca analizar si el estudio de los errores humanos en investigaciones de accidentes contribuye en las acciones para el control y reducción de accidentes de trabajo en una planta de sacrificios. El método de análisis utilizado fue la evaluación de los tipos de errores humanos a través de una secuencia de preguntas donde hay siempre dos opciones de respuestas -sí o no- y conforme el trazado de las respuestas se crea un algoritmo que permite la detección del error.

Los mayores porcentajes de errores fueron evidenciados en dos grupos, órdenes de los supervisores y procedimientos inadecuados. $\mathrm{Al}$ contrario al resultado encontrado en el estudio, las acciones de seguridad del trabajo están enfocadas en condiciones que no contribuyen en la prevención y control de los riesgos.

Se puede concluir que la herramienta de análisis de riesgos utilizada por la empresa, además de despreciar el análisis de los errores humanos, no soluciona de forma adecuada las causas ni las acciones levantadas en la investigación del accidente.

(Takeda F, Pereira A, Gonzales A, Monterrosa A, 2017. Fallas Humanas y Accidentes de Trabajo: Una Contribución de las Investigaciones en Prevención, Control de los Riesgos y Accidentes. Cienc Trab. MayAgo; 19 [59]: 120-127).

Palabras claves: SACRIFICIO DE ANIMALES, PREVENCIÓN DE ACCIDENTES, POSTURA.

\section{ABSTRACT}

In order to contribute to the control of industrial accidents this research seeks to analyze the study of human error in accident investigations contributes in actions for the control and reduction of accidents in a poultry slaughterhouse.

The method of analysis was used to assess the types of human errors through a series of questions in which there are always two choices of answers, with a yes or no and as the way of answers creates an algorithm for error detection.

The largest percentages of errors were found in two groups, the supervisor order and inadequate procedures. Contrary to the results found in the study, the work safety actions are focused on conditions that do not contribute to the prevention and control of risks.

It can be concluded that the risk analysis tool used by the company in addition to neglecting the analysis of human error, does not address properly the causes and actions raised in the investigation of the accident.

Key words: ANIMAL CULLING, ACCIDENT PREVENTION, POSTURE.

\section{INTRODUCCIÓN}

Las industrias de sacrificio y corte de pollos se destacan en el crecimiento productivo y en el crecimiento de indicadores en las enfermedades y accidentes relacionados al trabajo. ${ }^{1}$ Investigaciones en frigoríficos muestran que son numerosas las causas de la aparición de enfermedades y accidentes. ${ }^{2-10}$

\section{Correspondencia / Correspondence:}

Armando Monterrosa Quintero

Universidad Federal de Santa Catarina

Trindade, Caja Postal 476 CEP 88040-900

Florianópolis, Santa Catarina, Brasil

Tel.: 05548 9833-6029

e-mail: adomonterrosa@hotmail.com

Recibido: 25 de Abril de 2017 / Aceptado: 05 de Junio de 2017
En compensación, se percibe que los mecanismos preventivos existentes y las herramientas utilizadas en la identificación de los factores envueltos en el origen de los accidentes y enfermedades unidos al trabajo en plantas de sacrificio no parecen atender aún las demandas de las organizaciones y principalmente de los trabajadores. ${ }^{11}$

La historia de la seguridad del trabajo es rica en teorias que intentan explicar las causas de los accidentes ${ }^{12,13}$, pero no específica qué método debe ser utilizado para determinada empresa o accidente laboral.

Un punto relevante que debe ser observado respecto a los accidentes de trabajo es que a pesar de la existencia de las Normas de Seguridad en Brasil, que son obligatorias en todas las empresas que tienen trabajadores regularmente registrados, se observa que no siempre, de acuerdo con los informes de las causas de los accidentes, las orientaciones de las normas fueron seguidas. ${ }^{14}$ También es de conocimiento que gran proporción de los accidentes es atribuido a las fallas humanas, en cerca del 70 al $80 \%$ de los 
casos. ${ }^{15}$ Por lo tanto, decir que un accidente fue causado por una falla humana no es suficiente para explicar las causas del accidente en un nivel que pueda ser utilizado para el aprendizaje organizacional. Se debe recordar que la falla humana es la consecuencia y no la causa. ${ }^{15}$ Muchas veces los accidentes laborales pueden ser ocasionados por factores relacionados al ambiente de trabajo y por aspectos organizacionales. ${ }^{16}$

En el proceso de investigación de un accidente normalmente no se analiza todo lo que estaba envuelto en la situación que generó un accidente, pues en la mayoría de las veces se parte enjuiciando al involucrado, sobre el cual surgen posibles preguntas: ¿cómo puede haber olvidado esto?, ¿cómo no vio que eso podía causar un accidente ${ }^{17}$ Esta son otras condiciones que contribuyen negativamente en el proceso del análisis de las causas, atribuyendo en la mayoría de los casos a la falla del acidentado. ${ }^{17}$

La psicología, desde su origen, se interesó por el análisis de los errores humanos con sus primeras aplicaciones en intereses pedagógicos, de estudios relacionados a la atención, carga de trabajo y el éxito escolar. ${ }^{18}$

El objetivo de una investigación no es encontrar cuál fue el error del trabajador, pero sí comprender por qué las acciones y evaluaciones tienen un sentido en el momento en que ocurrió el accidente. ${ }^{17,21}$

Todas las posibles causas deben ser detectadas en la investigación del accidente para entenderlo. Complementando este concepto y resaltando que es peligroso aislar el término error humano cuando ocurre un accidente, pues este término, en la visión ergonómica contemporánea, hace necesario analizar en conjunto con todos los factores que contribuyen en la aparición del evento indeseado. ${ }^{22}$ El acontecimiento de accidentes ocasionados por errores humanos puede estar conectado a factores asociados a modelos de desempeño cognitivo. ${ }^{23}$ Así, se torna necesario en la interpretación de las causas el conocimiento del investigador acerca de los aspectos cognitivos del accidentado y que en la investigación del accidente sea incluido un análisis y clasificación de la naturaleza de los errores humanos. $^{23}$

Se percibe que hay necesidad de investigar y administrar las condiciones que pueden causar un accidente; de esta forma, esta investigación tiene como objetivo analizar si el estudio de errores humanos en investigaciones de accidentes contribuye en las acciones para el control y reducción de accidentes de trabajo en plantas de sacrificios localizado en la región de Santa Catarina (Brasil).

\section{METODOLOGÍA}

Siguiendo los conceptos metodológicos fue realizado un estudio de caso, transversal, mediante datos secundarios del análisis documental de investigaciones de accidentes de trabajo con informes relacionados a los impases laborales - CAT, ocurridos en el período de enero a septiembre de 2015, de una planta de sacrificio de pollos localizado en la región de Santa Catarina (Brasil).

En la investigación se utilizó el método inductivo, el cual partió de los conocimientos disponibles de los datos de investigaciones de accidentes para posterior formulación teórica de los conceptos que dieron soporte para alcanzar los objetivos propuestos. ${ }^{25}$

En cuanto al universo y muestra, la investigación fue realizada con datos de 14 investigaciones de accidentes de trabajo que ocurrieron con empleados de la empresa, fueron evaluados apenas los accidentes típicos, excluyendo de la evaluación los accidentes de trayecto y las enfermedades ocupacionales, debido a la falta de control de las informaciones en las investigaciones.

Para la recolección de los datos, fueron analizadas las planillas de investigación de accidentes de trabajo de la empresa y para su interpretación fueron evaluados cualitativamente, utilizando el modelo adaptado de Saurinet al. (2008) ${ }^{26}$, conforme a lo mostrado en la Tabla 1.

Tabla 1.

Preguntas para análisis del algoritmo de fallas humanas.

\begin{tabular}{|c|c|c|c|}
\hline \multirow{2}{*}{$\begin{array}{l}\text { Algo- } \\
\text { ritmo }\end{array}$} & \multirow{2}{*}{ Pregunta } & \multicolumn{2}{|c|}{ Opciones de respuesta-conclusión } \\
\hline & & No & Si \\
\hline 1 & $\begin{array}{l}\text { ¿El trabajador conocía los procedimientos } \\
\text { y/o fue entrenado para esta tarea? }\end{array}$ & 2 & 9 \\
\hline 2 & $\begin{array}{l}\text { ¿El procedimiento y/o entrenamiento } \\
\text { era adecuado/aplicable? }\end{array}$ & $\begin{array}{l}\text { No hubo error } \\
\text { del trabajador }\end{array}$ & 3 \\
\hline 3 & $\begin{array}{l}\text { ¿El procedimiento y/o entrenamiento } \\
\text { fue seguido? }\end{array}$ & 6 & 4 \\
\hline 4 & ¿Hubo falla del trabajador? & $\begin{array}{l}\text { No hubo error } \\
\text { del trabajador }\end{array}$ & 5 \\
\hline 5 & $\begin{array}{l}\text { ¿Era una situación nueva } \\
\text { o imprevista? }\end{array}$ & Deslice & $\begin{array}{l}\text { Error en el nivel } \\
\text { de conocimiento }\end{array}$ \\
\hline 6 & $\begin{array}{l}\text { Si el procedimiento fuera seguido, } \\
\text { ¿el incidente hubiera acontecido? }\end{array}$ & 7 & $\begin{array}{l}\text { No hubo error } \\
\text { del trabajador }\end{array}$ \\
\hline 7 & ¿Otro trabajador haria lo mismo? & 8 & $\begin{array}{l}\text { No hubo error } \\
\text { del trabajador }\end{array}$ \\
\hline 8 & ¿El error fue intencional? & Lapso de memoria & Violación \\
\hline 9 & $\begin{array}{l}\text { ¿El trabajador fue designado por su } \\
\text { supervisor para ejecutar esta tarea? }\end{array}$ & Violación & $\begin{array}{l}\text { No hubo error } \\
\text { del trabajador }\end{array}$ \\
\hline 10 & ¿Había otro trabajador involucrado? & 1 & Fin \\
\hline
\end{tabular}

El método presentado en la Tabla 1 es utilizado para identificar y clasificar los posibles tipos de errores humanos. ${ }^{26}$

Se puede observar en la Tabla 1 una secuencia de interrogaciones que inicia con la pregunta: ¿el trabajador conocía los procedimientos y/o fue entrenado para esta tarea?

Después de iniciar las preguntas, se verifica que para cada pregunta hay opción de dos respuestas, sí o no, donde la secuencia del algoritmo es dada conforme los caminos que llegan a la clasificación del motivo que contribuyó en la aparición del accidente. Por ejemplo:

- Respuesta de la primera pregunta: no - sigue para la pregunta 2;

- Respuesta de segunda pregunta: no - no hubo error del trabajador. El algoritmo para condición del ejemplo es: 1-2, en el cual no hubo error del trabajador en el accidente.

La investigación fue aprobada por el Comité de Ética e investigación de la Universidad Federal de Santa Catarina (UFSC) el 23 de noviembre del 2015 y hubo consentimiento de la empresa en realizar la investigación. En la investigación atiende la Resolución CNS0020466/12, de 12/09/2012 y complementarias.

\section{RESULTADOS}

Entre las investigaciones de accidentes analizadas, 21\% ocurrieron con trabajadoras del sexo femenino y $79 \%$ con trabajadores del sexo masculino. Con relación a la gravedad de los accidentes, que es medida en función de los dias retirados de los accidentados, $21 \%$ ocurrieron de 0 a 5 días de incapacidad, $8 \%$ ocurrieron de 6 a 10 días de incapacidad, 21\% ocurrieron de 11 a 15 días de incapacidad y 50\% ocurrieron con más de 15 días de incapacidad.

En resumen, con los resultados obtenidos es posible verificar que el mayor o número de accidentados se concentra en el sexo mas- 
culino y que 50\% de los accidentes pueden ser considerados graves debido al número de días de incapacidad de los accidentados.

\section{Análisis de los accidentes}

Conforme a la gravedad del accidente, supervisores de producción y CIPA -Comisión Interna de Prevención de Accidentes- participan de la investigación y decisión de las acciones a ser realizadas.

En el primer accidente, el breve relato fue que la funcionaria estaba realizando la actividad de clasificar "patas de pollo en el sector de escaldado y al obstruirse la máquina "alimentadora de proceso" la trabajadora colocó la mano dentro del equipo encendido para destrabarlo; al realizar esto, el $3^{\circ}$ dedo de la mano izquierda quedó atorado en la guillotina del equipo. La funcionaria se encontraba desempeñando una actividad que no era de su función específica.

Las acciones propuestas fueron:

- La máquina solamente podrá ser operada por empleados habilitados para esta actividad.

- Los supervisores deben informar la substitución de los empleados en la línea de producción.

- Realizar un levantamiento de todos los empleados del sector para verificar si existen empleados fuera de su función.

- Verificar con mantenimiento la posibilidad de bajar la barrera de seguridad para aumentar la distancia de la guillotina imposibilitando el alcance de los miembros superiores de los operadores de la máquina.

El resultado del algoritmo de errores humanos para este accidente es 1-9, lo que representa la posible aparición de que no hubo errores del trabajador debido a que la persona tuvo una orden de un superior para ejecutar la actividad, la cual ocasionó una fractura en la mano izquierda de la accidentada.

En el segundo accidente, el relato fue que el empleado estaba realizando la actividad de retirar jaulas con pollos del camión y, al retirar las jaulas, su dedo quedó atorado entre dos de estas jaulas. La principal causa detectada en la investigación del equipo de SST es considerar que las jaulas están mojadas y sucias con excrementos de los pollos, lo que las hace resbaladizas.

Las acciones propuestas fueron:

- Entrenar todos los empleados de la plataforma en la correcta forma de retirar jaulas con pollos del camión.

El resultado del algoritmo de los errores humanos para este accidente muestra que es 1-2, lo que representa la posible aparición de que no hubo error del trabajador debido a un procedimiento inadecuado necesario para ejecutar la actividad, el cual ocasionó una fractura en la mano izquierda del accidentado.

En el tercer accidente, el relato corto fue que el empleado sin licencia de conducción estaba realizando la actividad de transportar plataformas de madera con productos de la cámara de almacenamiento para la antesala utilizando un montacargas eléctrico, cuando la carga resbaló y prensó su pie izquierdo contra el pilar de protección. Las acciones propuestas fueron:

- Prohibir que empleados sin habilitación ni entrenamiento manejen los montacargas.

El resultado del algoritmo de errores humanos para este accidente es 1-9, lo que representa la posible aparición de que no hubo errores del trabajador debido a que la persona tuvo una orden de un superior para ejecutar la actividad, la cual ocasionó un trauma en el pie izquierdo del accidentado.

En el cuarto accidente, el breve relato fue que el empleado estaba realizando la actividad de cortar alas de pollo cuando el cuchillo escapó de su mano y, al caer, él intentó cogerlo, pero el cuchillo impactó contra la estera y perforó su mano derecha; se descubrió que el mango del cuchillo es muy resbaladizo.

Las acciones propuestas fueron:

- Realizar entrenamiento con todos los que utilizan cuchillo, para orientar el uso adecuado de este elemento.

El resultado del algoritmo de errores humanos para este accidente es 1-2-3-6-7-8, lo que representa la posible aparición de un lapso de memoria del empleado debido a un error no intencional, el cual ocasionó un corte en la mano derecha del accidentado.

En el quinto accidente, el breve relato fue que el empleado estaba realizando la actividad de revisar las patas de los pollos haciendo la retirada de la piel. Él estaba realizando la actividad cuando se desprendió un pedazo de la máquina y alcanzó su mano izquierda. La principal causa detectada en la investigación del grupo de SST es que el accidente ocurrió debido a la ruptura del equipo durante el proceso de producción.

Las acciones propuestas fueron:

- Reparar el equipamiento que se rompió.

- Realizar una evaluación general en la máquina.

El resultado del algoritmo de errores humanos para este accidente es 1-2-3-4, lo que representa la posible aparición de que no hubo errores del trabajador, porque el accidente fue debido a una falla en la máquina.

En el quinto accidente, el relato abreviado del empleado dice que él fue solicitado para realizar el mantenimiento en la correa del motor de la máquina "transportadora redler 28" que estaba dañada. Según el operador de cuadro de comando, el mecánico interventor avisó para apagar y mantener apagado, pues él iría a realizar el mantenimiento en el motor del "redler 28" que se encontraba parado. De acuerdo con el mecánico él mismo cambió la correa del motor; sin embargo, la cinta quedó suelta en la polea, porque no era la correa adecuada. Al verificar que estaba floja, el mecánico fue hasta el motor de la "redler 27", retiró la protección de la correa y fue a verificar la numeración de ella. Pero el mecánico no avisó al operador del cuadro de comando ni a ningún otro empleado y tampoco bloqueó ni desactivó el motor y, al pasar la mano sobre la correa para verificar la numeración, el motor fue accionado y dejó presa su mano derecha en la polea del motor. La principal causa detectada en la investigación del grupo de SST es que no hay programa de mantenimiento preventivo para evitar el daño de ninguno de los equipamientos, además de que los mismos no atienden los dispositivos de seguridad conforme NR-12 (parada de emergencia y llave de desconexión).

Las acciones propuestas fueron:

- Implementar programa de mantenimiento preventivo.

- Realizar análisis de riesgo y adecuar los equipamientos de acuerdo con la norma NR-12.

- Implementar programas de bloqueo de los equipamientos.

- Realizar entrenamiento para la concientización sobre los riesgos en intervenciones mecánicas en los equipamientos.

El resultado del algoritmo de errores humanos para este accidente es 1-2-3-6-7-8, lo que representa la posible aparición de que hubo violación del trabajador, debido a un procedimiento inadecuado, el 
cual ocasionó el trauma/aplastamiento en la mano derecha del accidentado.

En el séptimo accidente, de acuerdo con el breve relato del empleado, él estaba descargando un camión con maíz con la ayuda de otro empleado; al percibir que el nivel del vaciado del maíz disminuyó, verificaron que había un pedazo de madera en la boca del equipo en el interior de la máquina receptora de granos. Los dos intentaron retirar la madera por el paso del elevador que está al lado de la máquina que transporta los granos para los silos. Sin embargo, no consiguieron retirarla por la lateral. En seguida, el empleado entró en la máquina para retirar el pedazo de madera al momento que ocurría la descarga del camión con maíz. Al bajar el producto, el empleado se resbaló por la escalera y cayó sobre el maíz que estaba siendo vaciado por la salida de la máquina transportadora de granos. Inmediatamente, el empleado tuvo parte del cuerpo cubierto por el maíz. Fueron detectadas como principales causas en la investigación del grupo SST la falta de entrenamiento de los trabajadores, falta de adecuación de la estructura para implantar la norma de espacios confinados y falta de mantenimiento de las señalizaciones existentes.

Las acciones propuestas fueron:

- Realizar entrenamiento de 16 horas con todos los trabajadores y vigías.

- Mapear todos los espacios confinados.

- Señalizar los espacios confinados.

- Implantar sistema de bloqueo en todos los espacios confinados.

- Adquirir equipos para trabajo en espacio confinado.

El resultado del algoritmo de errores humanos para este accidente es 1-9, lo que representa la posible existencia de una violación por parte de los trabajadores debido a la realización de un procedimiento inadecuado.

En el octavo accidente, el relato resumido fue que el empleado, después de regresar del almuerzo, estaba descargando los camiones con cajas de carne de pollo listas para la venta; él mismo estaba utilizando una máquina carretilla eléctrica para movilizar palletes con mercadería. De acuerdo con el empleado, al maniobrar la carretilla, en el acto de realizar una maniobra con la máquina, la misma se deslizó y acabó prensando la mano izquierda contra la barra de hierro. La principal causa detectada en la investigación del grupo SST es que el empleado fue contratado para esta función sin la exigencia mínima de calificación (certificado de operación de montacargas/elevador); se resalta que este accidente tuvo la misma causa detectada en el accidente.

Las acciones propuestas fueron:

- Garantizar que solamente colaboradores autorizados realicen estas operaciones.

- Intensificar el DDO (diálogo diario operacional) hablando para todo el equipo al respecto del accidente ocurrido.

- Diseñar carnets de identificación conforme NR-11.

El resultado del algoritmo de yerros humanos para este accidente es 1-9, lo que representa la posibilidad de que no hubo error del trabajador debido a una orden superior en ejecutar la actividad, el cual ocasionó un trauma en la mano izquierda del accidentado.

En el noveno accidente, el relato fue que el empleado, después de regresar de su descanso, fue a operar el carro de leña que alimenta la caldera, como de rutina. Según el empleado, él encendió el carro de leña en el cuadro de comando; luego, él mismo encendió el carro en el ojal de movimiento, que está en el propio carro. El empleado relata que giró el rostro para verificar el lado, en el sentido opuesto al carro de leña e instantáneamente colocó su mano izquierda en la polea que se encontraba con la protección abierta en el momento del accidente. No supo informar quién abrió la protección (amarilla). La principal causa detectada en la investigación del equipo de SST es que el empleado debería haber informado a su supervisor que la protección se encontraba abierta.

Las acciones propuestas fueron:

- Mantener las protecciones en las partes móviles de las máquinas cerradas.

- No operar máquinas sin las protecciones obligatorias.

- Intensificar entrenamiento hablando a todo el equipo al respecto del accidente ocurrido.

- Entrenar todos los mecánicos para mantener las protecciones después de las intervenciones de mantenimiento.

- Realizar un inventario de todas las poleas de motores para identificar las posibilidades de riesgos de accidentes similares a lo ocurrido.

El resultado del algoritmo de errores humanos para este accidente es 1-2-3-6-7-8, lo que representa la posible aparición de un lapso de memoria del funcionario debido a un error no intencional, el cual ocasionó un trauma/laceración en la mano izquierda del accidentado.

En el décimo accidente, el relato es que, después de terminar sus actividades en el sector de colgado de los pollos, por vuelta de las $23 \mathrm{~h} 50 \mathrm{~min}$, el empleado recordó que había olvidado cerrar el registro que controla el flujo de agua en una máquina. Volvió al sector para cerrar el registro y al dirigirse a la salida del sector, dejó de utilizar la plataforma de acceso a la salida y ejecutó la maniobra de saltar, al realizar la maniobra, se deslizó y su pie derecho quedo sub-inmerso dentro del tanque de inmersión de las jaulas (dentro del tanque hay agua caliente en torno de $70{ }^{\circ} \mathrm{C}$ y contiene una leve concentración de soda cáustica diluida automáticamente en el agua). La principal causa detectada en la investigación del equipo de SST es la falta de protección en las laterales del tanque de inmersión de jaulas para impedir que las personas salten por encima del tanque.

Las acciones propuestas fueron:

- Instalar protección en las laterales del tanque de inmersión de las jaulas para evitar que los funcionarios pasen por encima.

- Realizar inspección en los horarios de pausas y salidas para realizar la concientización de los trabajadores.

- Intensificar los entrenamientos hablando para todo el equipo al respecto del accidente ocurrido.

- Advertir al profesional que debe realizar el procedimiento de utilizar la plataforma de acceso al sector del colgado.

El resultado del algoritmo de errores humanos para este accidente es 1-2-3-6-7-8, lo que representa la posible violación del trabajador debido a un error intencional de un procedimiento inadecuado, la cual ocasionó una quemadura en su pierna derecha.

En el décimo primer accidente, el relato es que el empleado estaba realizando una doble actividad en el proceso de distribuir un contenedor y una de las cajas estaba averiada y otro empleado, que estaba aguardando a su vez para realizar el proceso, fue hasta la máquina plastificadora para arreglar la caja; cuando regresó, arrojó la caja en el pallet que estaba siendo montando y alcanzó la mano del empleado que montaba el pallet. La principal causa detectada en la investigación del equipo de SST es que el funcionario que realizó el 
procedimiento incorrecto estaba trabajando en la empresa hacía dos días. Verificando que fue falta de entrenamiento y acompañamiento al realizar las actividades en el sector de salida del producto.

Las acciones propuestas fueron:

- Advertir al colaborador que realizó un procedimiento incorrecto de arrojar la caja.

- Realizar la supervisión y acompañamiento de los colaboradores que inician sus actividades.

- Intensificar los entrenamientos hablando a todo el equipo respecto al accidente ocurrido.

El resultado del algoritmo de errores humanos para este accidente es 1-2-3-4, lo que representa la posibilidad de que no hubo error del trabajador debido a la falta de entrenamiento.

En el décimo segundo accidente, el relato es que, al realizar el procedimiento de desatascar el tanque de aceite de la centrífuga, el empleado no realizó el procedimiento de cerrar el registro de vapor y fue a desatascar con la manguera de agua. Siendo este procedimiento inadecuado, debido a que el aceite en alta temperatura no puede tener contacto directo con agua. Con esto el aceite hirviendo subió por encima de la centrífuga y comenzó a salpicar, alcanzando al empleado. La principal causa detectada en la investigación del equipo de SST es que el empleado dejó de seguir el procedimiento de seguridad do PO (Procedimiento Operacional) de la centrífuga, que debería haber apagado el calentamiento del tanque, abrir el registro del vapor para calentar el lodo y limpiar la tubería.

Las acciones propuestas fueron:

- Realizar reciclaje del entrenamiento de la PO de operación de la centrífuga.

- Advertir al profesional por el acto inseguro, pues él mismo ya había sido orientado a no mezclar aceite caliente con agua.

- Realizar con todo el equipo de la FFO entrenamiento práctico y procedimientos de seguridad en cuanto a la operación y manejo de la centrífuga en los casos en los que se necesite desobstruir el depósito del aceite.

El resultado del algoritmo de errores humanos para este accidente es 1-2-3-6-7-8, que representa la posible aparición de un lapso de la memoria del empleado debido a un error no intencional.

En el décimo tercer accidente, el relato es que el empleado en la función de mecánico, junto con el supervisor de mantenimiento, estaba realizando la actividad de sustituir la boquilla de la ventana de inspección de seguridad de la caldera, pues el operador había identificado fugas de vapor por la boquilla. Durante esta actividad, el empleado inhaló vapores y humo que estaban escapando en el local de la intervención. La principal causa detectada en la investigación del equipo de SST es que la boquilla debería haber sido substituida de acuerdo con las orientaciones del fabricante.

Las acciones propuestas fueron:

- Verificar si está siendo realizada los cambios de las boquillas de las ventanas de inspección de seguridad de acuerdo con las orientaciones del fabricante;

- Realizar entrenamiento de seguridad para todos los colaboradores del sector de mantenimiento, referente al procedimiento de seguridad al realizar mantenimiento en la caldera e informar que tienen derecho de rechazar la acción cuando la actividad coloca el colaborador en riesgo.

- Verificar si el planeamiento de las acciones recomendadas por el ingeniero mecánico en las inspecciones de la caldera están siendo cumplidas.
- Realizar sensibilizaciones periódicas con temas de seguridad con los supervisores/coordinadores de la unidad.

El resultado del algoritmo de errores humanos para este accidente es 1-2-3-6-7, lo que representa la posibilidad de que no hubo error del trabajador debido a que fue una orden de su superior para ejecutar la actividad, lo cual ocasionó una intoxicación respiratoria para el accidentado.

En el décimo cuarto accidente, la historia dice que el empleado estaba realizando la actividad de descargar las jaulas de los pollos en el ascensor de jaulas del colgado y al llevar una jaula para la cinta transportadora terminó dejando su mano izquierda junto con la jaula que terminó golpeando su mano izquierda. La principal causa detectada en la investigación del equipo de SST es que las jaulas están mojadas y sucias con excrementos de los pollos, por lo mismo, están resbaladizas. Se resalta que este accidente tuvo una causa muy similar a la detectada en el accidente 2 que ocurrió 6 meses y 21 dias antes de este.

Las acciones propuestas fueron:

- Entrenar todos los colaboradores de la plataforma en la correcta retirada de las jaulas del camión.

El resultado del algoritmo de errores humanos para este accidente es 1-2, que representa la posibilidad de que no hubo error del trabajador, debido a un procedimiento inadecuado necesario para ejecutar la actividad.

\section{Discusión de los Resultados}

Primeramente son discutidos los resultados de los algoritmos de los errores humanos de todos los accidentes.

En el Gráfico 1 son mostrados los resultados de la compilación de los datos obtenidos en los algoritmos.

\section{Gráfico 1.}

Porcentaje de los tipos de errores.

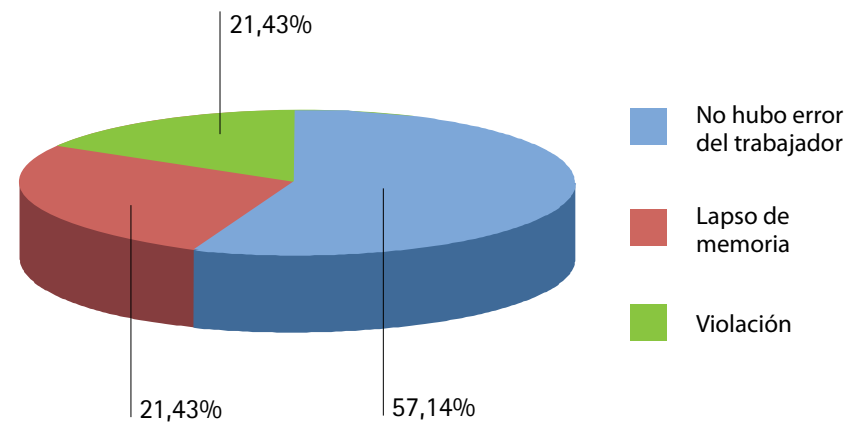

En el Gráfico 1 se verifica que del total de los casos analizado, el $57,14 \%$ resultó en que no hubo error del trabajador. Es decir, de acuerdo con el modelo de análisis utilizado, se puede afirmar que los errores no fueron causados directamente por los accidentados; por ejemplo, se cita que conforme el análisis de los datos, ocurrieron por orden del supervisor, por un procedimiento inadecuado de la empresa, por falta de entrenamiento, entre otros. Con este resultado es posible verificar que más de la mitad de las acciones no pueden estar enfocadas directamente en los trabajadores y sí en las condiciones de trabajo ofrecidas para ellos.

En seguida, con el mismo porcentaje de 21,43\% están el lapso de memoria y la violación, que encuadraron en las demás condiciones 
posibles de error en los accidentes analizados. Se resalta que los resultados obtenidos son de suma importancia para el tratamiento de las acciones que deben ser realizadas con el fin de evitar o reducir el número de errores que contribuyen a la aparición de accidentes. Con relación al lapso de memoria, que está relacionado a una condición cognitiva del trabajador, es decir, el comportamiento en el cual la acción no ocurre como es planeada ${ }^{12}$. Por lo tanto, esta es una condición que especificamente puede ser evaluada con la aplicación de la AET -Análisis Ergonómico del Trabajo-, que tiene como objetivo medir y describir las condiciones planeadas en el análisis de la tarea y de forma resumida comparar con las actividades realizadas en la fase del análisis de la actividad. Con los resultados detallados de los análisis de la tarea y de la actividad propuesta en la realización de la AET, se puede descubrir y/o medir las condiciones que contribuyen a la aparición del lapso de memoria.

Conforme los resultados referentes a los casos donde se identificó que hubo violación, que también obtuvo el resultado de 21,43\%, según los datos compilados, estos apuntan que en todos los casos fueron influenciados por la ejecución de procedimientos inadecuados por parte del empleado en la hora de realizar las actividades. En esta condición de violación para los casos analizados también es posible utilizar la AET para analizar y descubrir los motivos que llevan a violar los procedimientos establecidos por la empresa para ejecutar las actividades. Por lo tanto, específicamente en este caso presentado, la violación de los procedimientos no resultó en condiciones positivas para la empresa ni para los trabajadores.

Es importante resaltar que, de acuerdo con el método de definición del algoritmo, las condiciones de error a nivel de conocimiento también podrían ser la causa de las fallas que resultan en accidentes; sin embargo, no fueron evidenciados en ninguno de los casos de los accidentes.

En la secuencia de la discusión de los resultados, fueron analizadas las fallas que contribuyeron para la aparición del error. Los resultados son presentados en el Gráfico 2.

\section{Gráfico 2.}

Fallas detectadas.

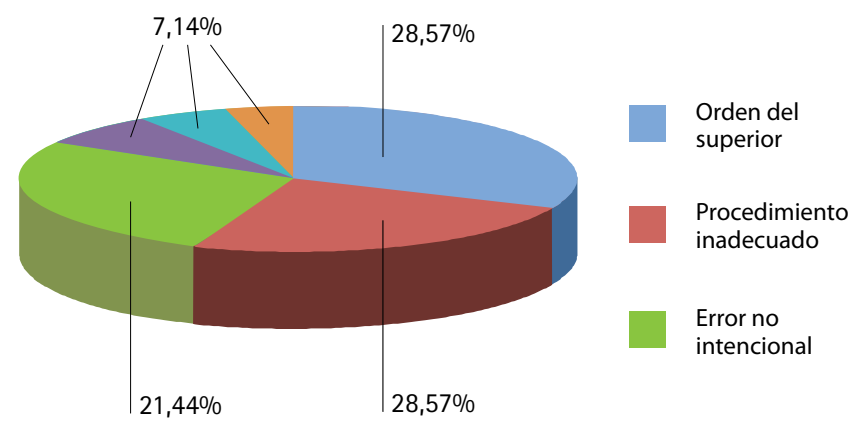

\section{Falta de entrenamiento}

Se percibe en los resultados del Gráfico 2 que los mayores porcentajes, 28,57\%, fueron evidenciados en dos grupos, orden del supervisor y procedimientos inadecuados, ambos sin relación directa con las acciones direccionadas a los trabajadores.

Es importante resaltar que conforme los datos del Gráfico 2, el error intencional y la falta de entrenamiento aparecen con los menores porcentajes de fallas, con apenas 7,14\% cada ítem. Con relación a la falta de entrenamiento, en este ítem fue evidenciado en el décimo primer accidente, donde este fue causado por el compañero de trabajo que no había recibido entrenamiento adecuado para la actividad a ser ejecutada. Es decir, se puede eliminar la condición de falta de entrenamiento, porque en este caso el accidente fue causado por un compañero de trabajo, caso contrario tendría una condición de cero en el caso con fallas detectadas por falta de entrenamiento.

Evidentemente, fue destacada esta condición de falta de entrenamiento debido a la lectura y estratificación de las acciones del equipo de SST presentadas en los resultados de la investigación. Las acciones del SST fueron estratificadas en los items:

- Acciones en el entrenamiento.

- Acciones en procedimientos operacionales.

- Acciones vinculadas a las máquinas.

- Acciones en errores intencionales de los trabajadores.

- Acciones en errores no intencionales de los trabajadores.

- Acciones para los supervisores.

En el Gráfico 3 son presentados los resultados de los datos recopilados de las acciones del SST de la empresa.

Gráfico 3.

Acciones del SST de la empresa.

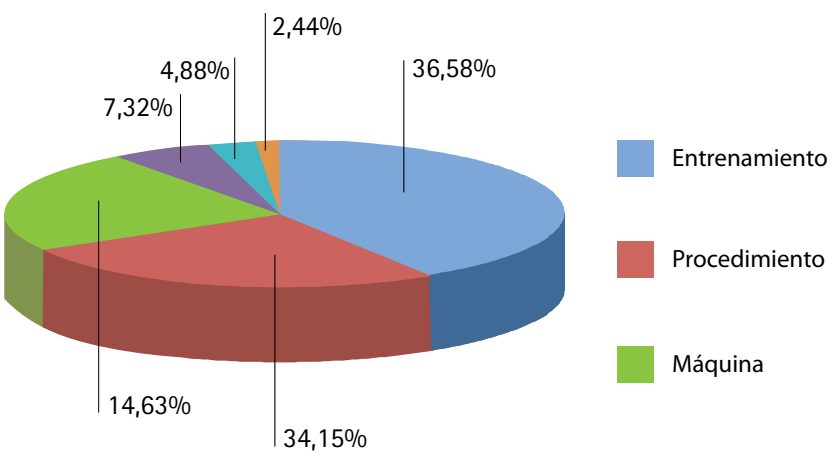

Se percibe en los resultados del Gráfico 3 que el mayor índice de las acciones del equipo de SST de la empresa se concentra en entrenamientos con un porcentaje de $36,58 \%$, seguido de procedimientos con un porcentaje de $34,15 \%$ de los casos estudiados. Se puede verificar que la falta de una metodología para el análisis del tipo de error y fallas detectadas conduce a los investigadores del equipo de SST a tomar acciones inversas a las condiciones que realmente causaron el error. Otro punto para destacar es que evidentemente se nota que en la mayoría de las acciones se atribuye la culpa a la falta de entrenamiento de los trabajadores.

En el penúltimo lugar en el ranking, tenemos las acciones para los supervisores, con 4,88\% de los casos. En este estudio se demuestra que en la metodología del análisis de los accidentes utilizada por la empresa está nuevamente invertida la prioridad de las acciones. Este caso en particular, debería estar en primer lugar debido al número de accidentes de trabajo que ocurrieron por causa de las órdenes de los supervisores, casos en los cuales los trabajadores no estaban preparados para lidiar con las situaciones de riesgo.

Ciertamente si las acciones que fueron realizadas son contrarias a las causadoras de los accidentes, los índices de accidentalidad se mantendrán o aumentarán en la proporción en la que ocurren. Tal condición puede ser destacada como por ejemplo en el tercer accidente relatado. Donde al leer los acontecimientos y las acciones tomadas, observamos que después de 2 meses y 4 días, ocurrió nuevamente otro accidente, el octavo, en condiciones totalmente 
similares, lo que indica que las acciones que fueron tomadas cuando ocurrió el accidente por primera vez no fueron realmente las adecuadas.

Un caso similar fue detectado en los accidentes 2 (accidente inicial) y 14 (se repitió el accidente 2 en condiciones muy similares) $\mathrm{y}$ al igual que en el ejemplo anterior, ambos accidentes tuvieron las mismas causas y fueron tomadas las mismas acciones. Cabe resaltar que en los accidentes 2 y 3, las acciones tomadas ya se encontraban como concluidas en el período que ocurrieron los primeros accidentes. Por lo tanto, los análisis nos ayudan a verificar que las acciones descritas sin la utilización de un análisis de los errores y fallas no eliminan o reducen la aparición de las mismas condiciones que contribuyeron a la aparición de accidentes que ya ocurrieron en la empresa.

Finalmente, es importante resaltar que el 50\% de los casos, es decir, 7 accidentes, fueron considerados graves, debido a que ocasionaron en los trabajadores lesiones en las que fueron necesarias más de 15 días de recuperación para el tratamiento de la lesión resultante del accidente, además 4 trabajadores aún se encuentran incapacitados en el sistema del INSS -Instituto Nacional del Seguro Social-, y que están con previsión de regreso solo en el 2016. En total, debido a los 14 accidentes fueron contabilizados, considerando las incapacidades, 1105 días perdidos hasta el día 31 de diciembre de 2015.

\section{CONCLUSIONES}

Según los resultados del algoritmo de errores humanos analizados con datos de las investigaciones de los accidentes, en más de la mitad de los accidentes fue identificado que no hay culpa de los trabajadores; por lo tanto, si no hay culpa, consecutivamente el foco de las acciones no puede ser priorizado en acciones vinculadas a los trabajadores.

Es posible notar que en las acciones de las investigaciones son limitadas, y hay un llamado "un valor muy alto" de los investigadores en atribuir la culpa de los accidentes a la falta de entrenamientos y procedimientos de los accidentados.

Inversamente a las causas y acciones de las investigaciones por parte de la empresa, los resultados de la investigación demuestran que, en primer lugar, están las fallas por parte de los supervisores y de procedimientos inadecuados, donde ambos presentan 28,7\% de las condiciones, sumando 57,4\% de las fallas detectadas; y, en las acciones del SST, los supervisores resultan apenas con el $4,88 \%$ de las acciones y los procedimientos inadecuados con el $34,15 \%$ de las acciones. Se nota que los procedimientos inadecuados tienden a un resultado próximo entre el algoritmo y acciones del SST. Por lo tanto, al destacar esta condición de entrenamiento evidenciado como en las acciones del SST con 36,58\% y en el resultado del algoritmo con apenas 7,14\%.

Se verifica que las acciones propuestas en las investigaciones son contrarias a las causas de errores cometidos en los accidentes de trabajo con relación a las condiciones cognitivas evidenciadas en los entrenamientos.

Los resultados también apuntan a que la herramienta de análisis de riesgos utilizada por la empresa, además de despreciar el análisis de los errores humanos, sea el causado por el accidentado o por algún factor externo al accidente, no soluciona de forma adecuada las causas levantadas en las investigaciones del accidente.

En este estudio el modelo de análisis de errores humanos no se vuelve un modelo padrón para las investigaciones de accidentes en mataderos y frigoríficos; por lo tanto, no hay cómo despreciar las investigaciones de accidentes porque no evalúan todos los posibles errores que pueden ocurrir durante el trabajo. Por lo tanto, además de un análisis del accidente, se debe tener en cuenta las posibles determinantes que actúan y contribuyen a la aparición de un accidente.

Se resalta que una o más condiciones de riesgo no solucionadas exponen a otros trabajadores a los accidentes que pueden acarrear daños a la salud, la integridad física, lesiones temporarias, permanentes o incapacidad y hasta la muerte. Como fueron analizadas apenas 14 investigaciones de accidentes relevantes del año 2015, no hay cómo afirmar que estos accidentes nunca ocurrieron antes. Evidentemente, ocurren resultados negativos de las acciones que no contribuyen en el efectivo control de la condición de riesgo, y también resultados negativos con trabajadores incapacitados con lesiones consideradas graves y trabajadores insatisfechos debido a la atribución de la culpa y despidos injustificados, es decir, advertencias debido a la atribución de la culpa. 
1. Marra GC, Souza $L H$, Cardoso TAO. Biossegurança no trabalho em frigorificos: da margem do lucro à margem da segurança. Ciênc saúde coletiva [online]. 2013[acesso Jun 2017];18(11):3259-3271. Disponivel em: http://www.scielo. $\mathrm{br} / \mathrm{pdf} / \mathrm{csc} / \mathrm{v} 18 \mathrm{n} 11 / 16 . \mathrm{pdf}$

2. Armstrong J, Buckle $P$, Fine L, Hagberg BJ, Kilbom A, Kuorinka IA, Silverstein A, Sjogaard G, Viikari-Juntura EA. Conceptual model for work-related neck and upper-limb musculoskeletal disorders. Scand J Work Environ Health. 1993;19(2):73-84.

3. Bao $S$, Silverstein $B$, Cohen $M$. As electromyography study in three high risk poultry processing jobs. Int J Ind Ergon. 2001;27(6):375-385.

4. Busnello GF, Dewes M. Doenças Osteomusculares Relacionadas à Atividade de Trabalhadores de Frigorificos de Frangos. Braz J Surg Clin Res. 2013; 4(3):27-32.

5. Frost $\mathrm{P}$, Andersen $\mathrm{J} H$, Nielsen VK. Occurrence of carpal tunnel syndrome among slaughterhouse workers. Scand J Work Environ Health. 1998; 24(4):285-292.

6. Heck FM. Uma Geografia da Degradação do Trabalho: 0 Adoecimento dos Trabalhadores em Frigorificos. Rev Percurso. 2013;5(1):3-31.

7. Juul-Kristernsen B, Fallentin N, Hansson GA. Physical workload during manual and mechanical deboning of poultry. Int J Ind Ergonom. 2002;29(2):107-115.

8. Musolin K, Ramsey JG, Wassel JT, Hard DL. Prevalence of carpal tunnel syndrome among employees at a poultry processing plant. Appl ergon. 2014;45(6):1377-1383.

9. Sommerich CM,Mcglothlin JD,Marras WS. Occupational risk factors associated with soft tissue disorders of the shoulder: a review of recent investigations in the literature. Ergonomics. 1993;36(6):697-717.

10. Sundstrup $E$, Jakobsen MD, Jay K, Brandt M, Andersen LL. High Intensity Physical Exercise and Pain in the Neck and Upper Limb among Slaughterhouse Workers: Cross-Sectional Study. BioMed Res Int, 2014(2014) ID 218546:00-05.

11. 7. Jakobi HR, Barbosa-Branco A, Bueno LF, Ferreira RGM, Camargo LMA. Benefícios auxílio-doença concedidos aos trabalhadores empregados no ramo de carne e pescado no Brasil em 2008. Cad Saúde Pública. 2015;31(1):194-207.
12. Binder MCP, Almeida IM. Estudo de caso de dois acidentes do trabalho investigados com o método de árvore de causas. Cad Saúde Pública. 1997;13(4):749-760.

13. Guimarães LBM, Costella MF. Segurança no Trabalho: Acidentes, Cargas e Custos Humanos. In: Guimarães LBM. Ergonomia de Processos. Vol. 2. 4 ed. Porto Alegre: PPGEP-UFRGS; 2006.

14. Rodrigues MK, Godoy LP, Lorenzett DB, Guarienti EP, Portela OT, Rodrigues LK. Abordagem em Saúde e Segurança do Trabalho: Estudo do Perfil dos Servidores de um Hospital Universitário. Espacios. 2013;34(7):1-4.

15. Reason J. Human error. Cambridge: Cambridge University Press; 1990.

16. Reason J. Combating omission errors through task analysis and good reminders. Qual Saf Health Care. 2002;11(1):40-44.

17. Dekker S. The field guide to human error investigations. London: Ashgate; 2002.

18. Falzon P. Ergonomia. São Paulo: Blucher; 2007.

19. Groeneweg J. Hazard Analysis: The accident causation model. In: Stellman JM, editor. The ILO Encyclopaedia of Occupational Health and Safety.4th ed. Geneva: ILO; 1998.

20. Woods D, Johannese $L$, Cock $R$, Sarter NB. State-of-the-art-report: Behind Human Error: cognitive Systens, computers, and hindsight. Columbus: CSERIAC; 1994.

21. Costela LGC. Contribuições para aperfeiçoamento em um método de classificação de tipos de erros humanos com base na investigação de acidentes na construção civil (dissertação). Curso de Pós Graduação em Engenharia de Produção, Programa de Pós Graduação em Engenharia de Produção. Porto Alegre: Universidade Federal do Rio Grande do Sul; 2009. 82 p.

22. Vidal MC. Ergonomia Cognitiva. Apostila do Curso de Especialização Superior em Ergonomia. Rio de Janeiro: Fundação COPPETEC-COPPE-UFRJ; 2003.

23. Amalberti R. La conduite des sistèmes à risques. Paris: Presses Universitaires de France; 1996. (Le Travail Humain.)

24. Lakatos EM, Marconi MA. Técnicas de Pesquisa. São Paulo: Atlas; 2007.

25. Gil, AC. Como Elaborar Projetos de Pesquisa. São Paulo: Atlas; 1989.

26. Saurin $T A$, Guimarães $L B M, C o s t e l l a ~ M F$, Ballardin L. An algorithm for classifying error types of front-line workers based on the SRK frame work. International J Ind Ergonom. 2008;38(11-12):1067-1077. 